\title{
Retinite idiopática, vasculite, aneurismas e neurorretinite (IRVAN): relato de caso
}

\author{
Idiopathic retinal, vasculitis, aneurysms and neuroretinitis (IRVAN): case report
}

\author{
Kelly Fernandes de Paula Rodrigues ${ }^{1}$, Verônica Castro Lima², Tiago Eugênio Faria e Arantes ${ }^{1}$, Kimble Teixeira Fonseca Matos ${ }^{1}$, Cristina Muccioli ${ }^{1}$
}

\section{RESUMO}

Relatamos caso de um paciente de 14 anos, sexo masculino, que foi admitido com queixa de embaçamento visual bilateral há dois anos. Ao exame oftalmológico observou-se leve hiperemia e edema de disco óptico bilateral, exsudação retiniana, poucas hemorragias retinianas, múltiplos aneurismas, assim como sinais de vasculite. A angiofluoresceinografia demonstrou isquemia periférica extensa, dilatações e hiperfluorescência das paredes dos vasos, e vazamento tardio do disco óptico nas fases finais do exame em ambos os olhos. Este caso representa uma rara entidade caracterizada por oclusão retiniana vascular periférica, vasculite retiniana, múltiplos aneurismas retinianos e neurorretinite (IRVAN). Avaliação sistêmica e laboratorial não revelaram nenhuma anormalidade. O paciente foi submetido à panfotocoagulação de retina com laser de argônio em ambos os olhos, e iniciado tratamento com prednisona via oral, com manutenção da acuidade visual de 20/25 depois de um ano de acompanhamento. O tratamento com laser deve ser considerado quando houver qualquer evidência angiográfica de má perfusão retiniana, eantes do desenvolvimento de qualquer sinal de neovascularização de retina.

Descritores: Uveíte; Retinite/diagnóstico; Aneurisma/diagnóstico;Vasculite retiniana/ diagnóstico; Angiofluoresceinografia; Glucocorticoides/uso terapêutico; Síndrome; Humanos; Masculino; Adolescente; Relato de casos

\begin{abstract}
A case of a 14 year-old boy that was admitted complaining of bilateral ocular visual blurring for 2 years is reported. The ophthalmological examination disclosed bilateral mild optic disc hyperemia and swelling, retinal exudation, few retinal hemorrhages, multiple aneurysms, as well as vasculitis. Fluorescein angiography showed extensive peripheral retinal ischemia, dilatations and hyperfluorescence of the vessels walls, and leakage of the optic disc in the late phases in both eyes. This rare case represents an entity characterized by peripheral retinal vascular occlusion, retinal vasculitis, multiple posterior retinal aneurysms, and neuroretinitis (IRVAN). Systemic evaluation and laboratory work-up did not suggest any systemic abnormality. Panretinal laser photocoagulation was performed in both eyes, and the patient was treated with oral prednisone with maintenance of the visual acuity after 1 year of follow-up. Laser treatment should be considered when angiographic evidence of widespread retinal no perfusion is present, and before the development of signs of retinal neovascularization.
\end{abstract}

Keywords: Uveitis; Retinitis/diagnosis; Aneurysm/diagnosis; Retinal vasculitis/diagnosis; Fluorescein angiography; Glucocorticoids/therapeutic use; Syndrome; Humans; Male; Adolescent; Case reports

\section{INTRODUÇÃO}

O diagnóstico da síndrome caracterizada por vasculite retiniana idiopática, aneurismas e neurorretinite (IRVAN) é baseado na presença de diversos achados clínicos. Três critérios maiores (vasculite retiniana, dilatações aneurismáticas nas bifurcações arteriais e neurorretinite), e 3 critérios menores (não perfusão capilar periférica, neovascularização de retina e exsudação macular) já foram bem definidos e são utilizados para auxiliar no diagnóstico de IRVAN(1). Esta é uma rara entidade clínica cujas alterações típicas já foram bem caracterizadas e seu estadiamento clínico já proposto anteriormente ${ }^{(1-4)}$. Inicialmente, acreditava-se ser uma condição autolimitada e benigna, entretanto, hoje se sabe que esta condição pode levar à perda visual grave quando não devidamente diagnosticada e tratada ${ }^{(1)}$.

Neste artigo relatamos o caso de um paciente do sexo masculino, 14 anos, com diagnóstico de IRVAN e que foi submetido precocemente ao tratamento clínico, obtendo manutenção da acuidade visual ao longo do seguimento.

\section{RELATO DE CASO}

Paciente de 14 anos, masculino, branco, solteiro, natural e procedente de São Paulo, compareceu ao setor de Uveítes/AIDS do De- partamento de Oftalmologia da Universidade Federal de São Paulo queixando-se de múltiplos episódios de turvação visual intermitente em ambos os olhos há cerca de dois anos. Ao realizar um exame de rotina há dois meses, foi evidenciado edema de disco óptico bilateral. 0 paciente negava antecedentes pessoais, familiares e oculares, assim como negava a presença de queixas sistêmicas. A acuidade visual melhor corrigida era 20/25 no olho direito e 20/25 no olho esquerdo. Ao exame de biomicroscopia, observava-se conjuntiva clara, córnea e cristalino transparentes, ausência de células ou flare na câmara anterior de ambos os olhos, porém apresentava 2+ de células no vítreo anterior bilateralmente. A pressão intraocular era de $14 \mathrm{mmHg}$ e o exame de gonioscopia mostrava ângulo aberto (sendo visto até a banda do corpo ciliar) em ambos os olhos. O exame de fundoscopia evidenciava nervo óptico hiperemiado e edemaciado bilateralmente, com contornos mal definidos e presença de exsudação lipídica peridiscal. Além disso, observava-se embainhamento vascular difuso nos 2 olhos associado a dilatações aneurismáticas em veias e artérias, e áreas extensas de não perfusão na periferia.

Os exames foram solicitados. Dentre estes angiofluoresceinografia (AFG), tomografia de coerência óptica (OCT), além de exames laboratoriais para realizar extensa investigação clínica quanto a doenças reumatológicas, inflamatórias e infecciosas. Encontramos: soroUNIFESP.

${ }^{1}$ Médico, Setor de Úvea/AIDS, Universidade Federal de São Paulo - UNIFESP - São Paulo (SP), Brasil. 
logias IgG positivas e IgM negativas para citomegalovírus e herpes simples; anti-HBs positivo, indicativo de vacinação prévia, e demais sorologias para sífilis (VDRL e FTA-Abs), hepatite C, anti-HIV negativas. Os demais exames solicitados estavam dentro da normalidade (hemograma, PPD, proteína C reativa, velocidade de hemossedimentação, eletroforese de hemoglobina, urina I, tomografia de tórax, uréia, creatinina, função e perfil hepáticos). Uma avaliação da reumatologia pediátrica também foi solicitada e não sugeriu nenhuma anormalidade (FAN, FR, antiDNA nativo, ENA - antiRo, antiLa, antiSm - negativos). O paciente não apresentava manifestação clínica ou queixas compatíveis com qualquer doença sistêmica cujo diagnóstico é predominantemente clínico; como também história epidemiológica para doenças sexualmente transmissíveis, contatos com animais e viagens. O exame de AFG evidenciou áreas extensas de isquemia retiniana em ambos os olhos, presença de sinais de vasculite difusa, e extravasamento de contraste na cabeça do nervo óptico nas fases tardias do exame, sendo este mais intenso no olho esquerdo (Figura 1). O OCT apresentou um espessamento da hialóide no olho esquerdo, sem outras alterações, que se manteve durante todo o seguimento.

Devido à presença de vasculite retiniana idiopática, dilatações aneurismáticas e neurorretinite, sem alterações sistêmicas associadas, o diagnóstico de IRVAN foi sugerido. Como o paciente apresentava sinais de inflamação, prednisona oral $40 \mathrm{mg} /$ dia em esquema regressivo lento foi iniciado, com redução de $5 \mathrm{mg} / \mathrm{semana}$ até ser atingido $10 \mathrm{mg}$ e após, de acordo com a resposta do paciente, 2,5 mg a cada 10 dias. Além disso, foi iniciado a fotocoagulação de retina com laser de argônio para o tratamento das regiões de isquemia, baseado na AGF. Após o tratamento, houve melhora do quadro inflamatório, que também pode ser observado na AGF (Figura 2).

O paciente evoluiu com muitos efeitos colaterais e intolerância ao corticoide quando em uso de $20 \mathrm{mg} /$ dia, por esse motivo uma droga imunossupressora (metotrexato $15 \mathrm{mg} / \mathrm{semana}$ ) foi associada ao tratamento. O mesmo está sendo acompanhado regularmente e apresenta quadro clínico estável após um ano de seguimento, ainda em uso do imunossupressor.

\section{DISCUSSÃO}

A associação clínica entre macroaneurismas e vasculite retiniana, predominantemente arterial, foi inicialmente descrita por alguns autores em $1983^{(5)}$.
A síndrome de IRVAN foi primeiramente descrita em 1995 em uma série de 10 pacientes ${ }^{(1)}$ e o conjunto único de achados que compõem essa rara entidade clínica fez com que esta fosse mais amplamente reconhecida entre os especialistas. Como a fisiopatogenia da doença envolve a vascularização da retina, o prognóstico depende do início precoce do tratamento da isquemia retiniana e de suas complicações ${ }^{(2)}$. Sabe-se que quanto mais precocemente o tratamento das áreas isquêmicas for instituído, menor será a taxa de progressão da doença e melhores serão os resultados para acuidade visual a longo prazo ${ }^{(2)}$. Além das complicações neovasculares, como hemorragia vítrea e glaucoma, a maculopatia exsudativa é uma das principais causas de baixa acuidade visual nestes pacientes.

Uma classificação baseada no grau de isquemia retiniana e suas sequelas foi proposta por Samuel et al. ${ }^{(2)}$, e divide a doença em 5 estágios (Tabela 1).

Os mesmos autores concluíram que o início da panfotocoagulação de retina antes ou logo após o desenvolvimento de qualquer neovascularização pode reduzir o risco de progressão da doença e perda visual grave ${ }^{(2)}$. Na mesma série de casos, pacientes no estágio 3 da doença apresentaram altas taxas de complicações neovasculares, apesar do tratamento com laser, e pacientes nos estágios 4 e 5 apresentaram prognóstico visual ruim, o que sugere a grande dificuldade em controlar as complicações neovasculares da doença. Por apresentar áreas de isquemia $360^{\circ} \mathrm{em}$ ambos os olhos, o nosso paciente se classifica como estágio 2, sendo portanto realizado tratamento com panfotocoagulação retiniana.

A lista de diagnósticos diferenciais de IRVAN inclui uma série de doenças vasculares, inflamatórias e infecciosas. Em casos relatados previamente, nenhuma associação com doenças sistêmicas foi identificada ${ }^{(2)}$. Entretanto, anamnese e interrogatório sistemático cuidadosos devem ser realizados em todos os casos para que sejam solicitados avaliação médica e exames laboratoriais direcionados, se necessário. Em nosso caso, avaliamos exaustivamente o paciente, não sendo encontrada nenhuma manifestação clínica compatível com qualquer doença sistêmica.

O papel do uso dos corticosteroides no tratamento do IRVAN ainda permanece controverso. Apesar da existência de células inflamatórias na câmara anterior ou vítreo corroborar com a etiologia inflamatória da doença, essas drogas não têm mostrado redução das áreas de vasculite ou de neovascularização a longo prazo. Em nosso
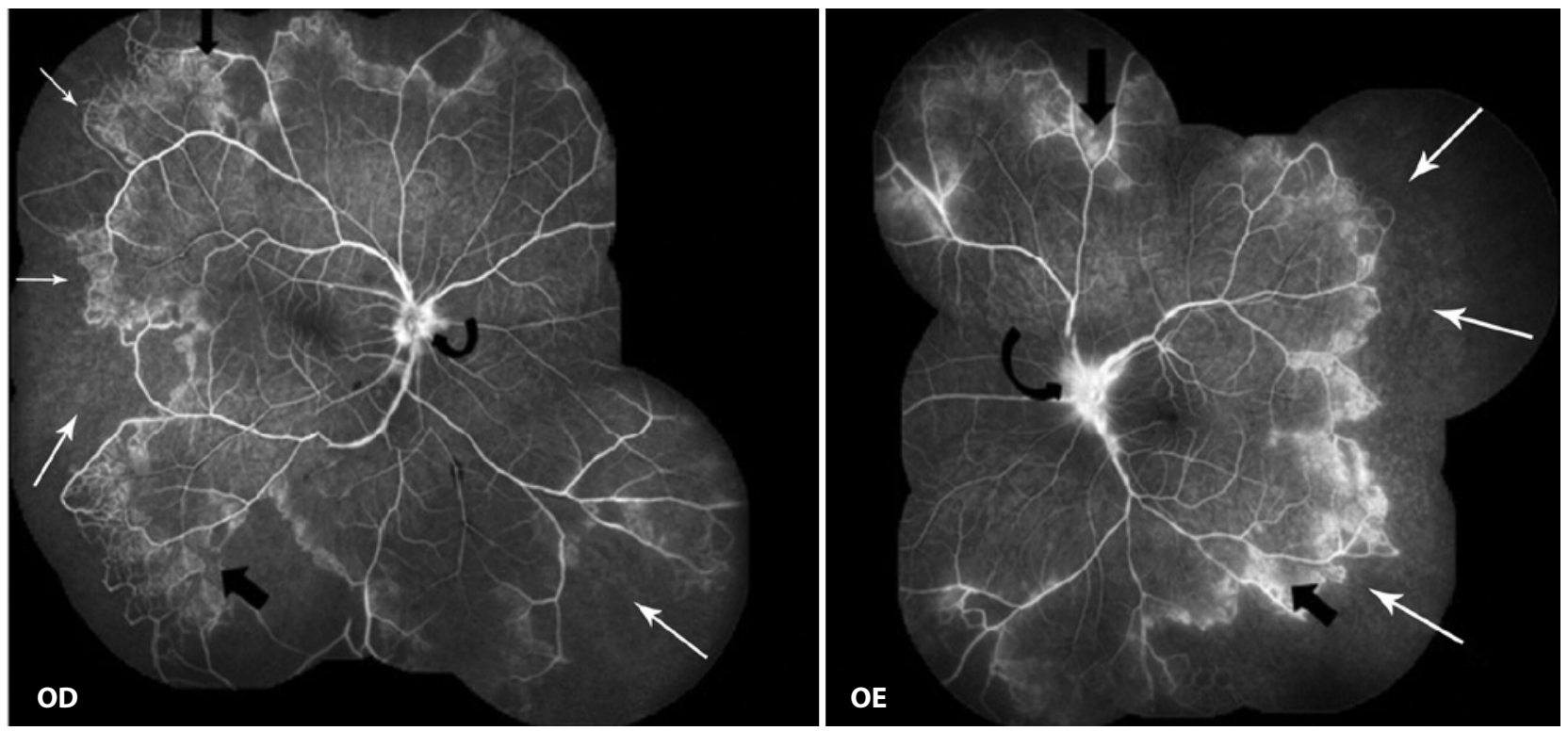

Figura 1. Montagem da angiofluoresceinografia, podendo-se observar áreas extensas de isquemia retiniana nos olhos direito (OD) e esquerdo (OE) (setas brancas finas), presença de sinais de vasculite difusa (setas pretas grossas), e extravasamento de contraste na cabeça do nervo óptico (setas pretas curvas) nas fases tardias do exame, sendo este mais intenso no $\mathrm{OE}$. 

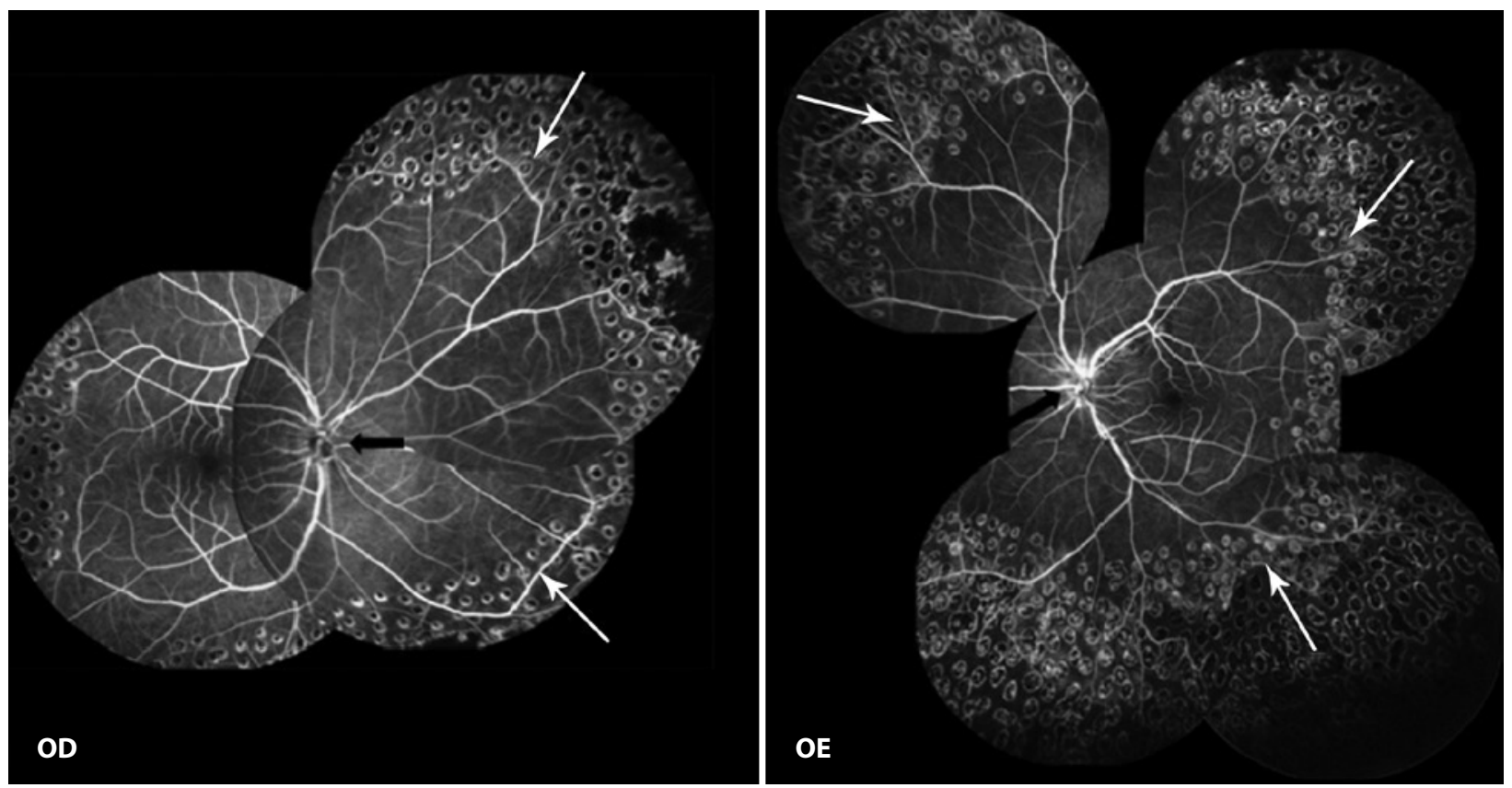

Figura 2. Angiofluoresceinografia após tratamento nos olhos direito (OD) e esquerdo (OE), apresentando redução da vitreíte, das áreas de vasculites (setas brancas finas) e do extravasamento do disco de ambos os olhos (setas pretas grossas).

Tabela 1. Classificação baseada no grau de isquemia retiniana e suas sequelas, proposta por Samuel et al. ${ }^{(2)}$

\begin{tabular}{ll}
\hline Estágios & Manifestações clínicas \\
\hline Estágio 1 & Macroaneurisma, exsudação, neurorretinite e vasculite retiniana; \\
Estágio 2 & Não perfusão capilar (evidência angiográfica); \\
Estágio 3 & $\begin{array}{l}\text { Neovascularização do segmento posterior, do disco ou de outra } \\
\text { região, e/ou hemorragia vítrea; }\end{array}$ \\
Estágio 4 & $\begin{array}{l}\text { Neovascularização do segmento anterior (rubeosis iridis); } \\
\text { Estágio } 5\end{array}$ \\
\hline
\end{tabular}

caso, resolvemos acrescentar prednisona, pela presença de sinais inflamatórios e acreditamos que apesar de algumas áreas de vasculite terem persistido após um ano de acompanhamento, houve redução da vitreíte, das áreas de vasculite e da hiperemia de disco em ambos os olhos.

A função da terapia imunossupressora ainda não é totalmente esclarecida, já que apenas poucos pacientes com a doença são tratados com ciclosporina ou metotrexato ${ }^{(6)}$, como também pela raridade do caso. Nosso paciente apresentou intolerância à corticoterapia, com diversos efeitos colaterais, dentre eles ganho de peso, labilidade emocional e estrias. Por esse motivo, optou-se por introduzir uma droga imunossupessora para que o corticoide fosse reduzido gradativamente. Quanto às injeções intravítreas, apesar de serem uma opção, preferimos fazer uso da terapia imunossupressora por ser um quadro bilateral e crônico, como também o paciente muito jovem, tentandose evitar, dessa maneira, o desenvolvimento de complicações.

O paciente descrito neste caso apresentou uma boa resposta à panfotocoagulação retiniana, com regressão das áreas de vasculite e hemorragias e redução do vazamento ao redor do disco detectado pela AFG. O corticoide foi introduzido devido à presença de sinais inflamatórios e, posteriormente, devido à intolerância medicamentosa o imunossupressor foi associado, com estabilização do quadro. É importante salientar a importância do tratamento precoce com fotocoagulação de retina nas áreas não perfundidas. Além disso, o papel do corticóide e do imunossupressor necessitam de melhor avaliação em estudos prospectivos a longo prazo, o que traz bastante relevância ao caso, já que houve uma boa resposta ao metotrexate, sempre lembrando da raridade da doença e devido a isso, da impossibilidade de estudos de maior extensão quanto à resposta das medicações referidas. Por corresponder a uma condição vascular retiniana rara, IRVAN deve ser reconhecida rapidamente e tratada adequadamente para que não haja perda visual bilateral grave.

\section{REFERÊNCIAS}

1. Chang TS, Aylward GW, Davis JL, Mieler WF, Oliver GL, Maberley AL, et al. Idiopathic retinal vasculitis, aneurysms, and neuro-retinitis: Retinal Vasculitis Study. Ophthalmology. 1995;102(7):1089-97.

2. Samuel MA, Equi M.A, Chang TS, Mieler W, Jampol LM, Hay D, et al. Idiopathic retinitis, vasculitis, aneurysms, and neuroretinitis (IRVAN): new observations and a proposed staging system. Ophthalmology. 2007;114(8):1526-9.

3. Tomita M, Matsubara T, Yamada H, Takahashi K, Nishimura T, Sho K, et al. Long term follow up in a case of successfully treated idiopathic retinal vasculitis, aneurysms, and neuroretinitis (IRVAN). Br J Ophthalmol. 2004;88(2):302-3.

4. Gedik S, Yilmaz G, Akça A, Akova YA. An atypical case of idiopathic retinal vasculitis, aneurysms, and neuroretinitis (IRVAN) syndrome. Eye. 2005;19(4):469-89.

5. Kincaid J, Schatz $\mathrm{H}$. Bilateral retinal arteritis with multiple aneurysmal dilatations Retina. 1983;3(3):171-8

6. Gonzales S, Rampini A, Rubino P, Mora P, Orsoni JG. A case of bilateral idiopathic retinitis, vasculitis, aneurysms and neuroretinitis (IRVAN) treated with discontinuous immunosuppressive therapy. Acta Ophthalmol. 2010;88 Suppl: S246. 